ANNALES

POLONICI MATHEMATICI

XXIX (1974)

\title{
Sur un système d'équations fonctionnelles lié au rapport anharmonique
}

\author{
par S. GołąB (Kraków)
}

Résnmé. Le but de cette Note est de caractériser d'une manière axiomatique le rapport anharmonique à l'aide d'un système d'équations fonctionnelles, notamment

$$
\begin{aligned}
& \sigma\left(x_{3}, x_{4}, x_{1}, x_{2}\right)-\sigma\left(x_{1}, x_{2}, x_{3}, x_{4}\right)=0, \\
& \sigma\left(x_{1}, x_{3}, x_{2}, x_{4}\right)+\sigma\left(x_{1}, x_{2}, x_{3}, x_{4}\right)=1, \\
& \sigma\left(x_{1}, x_{2}, x_{3}, x_{4}\right) \cdot \sigma\left(x_{1}, x_{2}, x_{4}, x_{5}\right)=\sigma\left(x_{1}, x_{2}, x_{3}, x_{5}\right) .
\end{aligned}
$$

On peut déduire de ce système sans aucune hypothèse de régularité de la fonction $\sigma$ la formule suivante pour la fonction cherchée $\sigma$ :

$$
\sigma\left(x_{1}, x_{2}, x_{3}, x_{4}\right)=\frac{\left[a\left(x_{1}\right)-a\left(x_{3}\right)\right]\left[a\left(x_{2}\right)-a\left(x_{4}\right)\right]}{\mid\left[a\left(x_{2}\right)-a\left(x_{3}\right)\right]\left[a\left(x_{1}\right)-a\left(x_{4}\right)\right]}
$$

où $a$ est une fonction inversible d'une variable réelle. Afin d'obtenir une solution unique du système (*) qui sera le rapport anharmonique classique, il suffit d'admettre les deux hypothèses additionnelles suivantes:

$1^{\circ}$ a. est une fonction strictement monotone,

$2^{\circ} \sigma\left(x_{1}, x_{2}, x_{3}, x_{4}\right)=-1$ pour la famille à deux paramètres des quadruples harmoniques de la forme $\left(x, y, \frac{2 x y}{x+y}, 0\right)$.

On sait que le rapport anharmonique peut être défini d'une façon axiomatique comme la solution d'une équation fonctionnelle [2]. A co problème ont été consacrés quelques travaux ultérieurs [3], [4], [5].

Dans le présent travail nous allons définir le rapport anharmonique $\Sigma\left(P_{1}, P_{2}, P_{3}, P_{4}\right)$ d'un quadruple de points colinéarie comme la solution d'un système d'équations fonctionnelles. Ces équations représentent certaines propriétés bien connues du rapport anharmonique.

Nous partons du fait que le rapport anharmonique $\Sigma\left(P_{1}, P_{2}, P_{3}, P_{4}\right)$ d'un quadruple de points $P_{i}(i=1,2,3,4)$ se transforme d'après les formules suivantes, si l'on effectue une permutation de la suite $P_{1}, P_{2}, P_{3}, P_{4}$ :

$$
\begin{aligned}
& \Sigma\left(P_{3}, P_{4}, P_{1}, P_{2}\right)=\Sigma\left(P_{1}, P_{2}, P_{3}, P_{4}\right), \\
& \Sigma\left(P_{2}, P_{1}, P_{3}, P_{4}\right)=\Sigma^{-1}\left(P_{1}, P_{2}, P_{3}, P_{4}\right), \\
& \Sigma\left(P_{1}, P_{3}, P_{2}, P_{4}\right)=1-\Sigma\left(P_{1}, P_{2}, P_{3}, P_{4}\right),
\end{aligned}
$$


Il suit de ces propriétés que $\Sigma$ peut prendre au plus 6 valeurs différentes dans des permutations arbitraires des points $\boldsymbol{P}_{\boldsymbol{i}}$.

Les relations (*) représentent en réalité les équations fonctionnelles pour la fonction $\Sigma$. Ces équations peuvent être transformées en équations fonctionnelles d'une fonction réelle $\sigma$ de quatre variables réelles indépendantes $x_{i}$ si l'on introduit les coordonnées affines des points $P_{i}$ :

$$
\sigma\left(x_{1}, x_{2}, x_{3}, x_{4}\right) \text {. }
$$

La résolution générale du système

$$
\begin{array}{r}
\sigma\left(x_{3}, x_{4}, x_{1}, x_{2}\right)-\sigma\left(x_{1}, x_{2}, x_{3}, x_{4}\right)=0, \\
\sigma\left(x_{2}, x_{1}, x_{3}, x_{4}\right) \cdot \sigma\left(x_{1}, x_{2}, x_{3}, x_{4}\right)=1, \\
\sigma\left(x_{1}, x_{3}, x_{2}, x_{4}\right)+\sigma\left(x_{1}, x_{2}, x_{3}, x_{4}\right)=1,
\end{array}
$$

ne présente pas de difficultés, elle est même presque triviale.

Pour obtenir cette solution il suffit de déterminer les valeurs de $\sigma$ tout à fait arbitrairement dans le domaine:

$$
D_{0} \stackrel{\text { def }}{=}\left\{\left(x_{1}, x_{2}, x_{3}, x_{4}\right) \mid x_{1}<x_{2}<x_{3}<x_{4}\right\}
$$

et de prolonger les valeurs de $\sigma$ au moyen des relations (1), (2), (3) sur le domaine $D$ tout entier, où $D \subset R^{4}$ est défini par la formule:

$$
D \stackrel{\text { def }}{=}\left\{\left(x_{1}, x_{2}, x_{3}, x_{4}\right) \mid x_{i} \neq x_{j} \text { pour } i \neq j\right\} \text {. }
$$

Une des conséquences de la définition classique du rapport anharmonique est la relation suivante:

$$
\sigma\left(x_{1}, x_{2}, x_{3}, x_{4}\right) \cdot \sigma\left(x_{1}, x_{2}, x_{4}, x_{5}\right)=\sigma\left(x_{1}, x_{2}, x_{3}, x_{5}\right) .
$$

Au lieu des relations $(1,2,3)$ nous admettrons les relations (1), (3) et (6) comme définition axiomatique du rapport anharmonique, c'està-dire nous considérons le système suivant d'équations fonctionnelle pour la fonction cherchée:

$$
\begin{aligned}
& \sigma\left(x_{3}, x_{4}, x_{1}, x_{2}\right)-\sigma\left(x_{1}, x_{2}, x_{3}, x_{4}\right)=0, \\
& \sigma\left(x_{1}, x_{3}, x_{2}, x_{4}\right)+\sigma\left(x_{1}, x_{2}, x_{3}, x_{4}\right)=1, \\
& \sigma\left(x_{1}, x_{2}, x_{3}, x_{4}\right) \cdot \sigma\left(x_{1}, x_{2}, x_{4}, x_{5}\right)=\sigma\left(x_{1}, x_{2}, x_{3}, x_{5}\right) .
\end{aligned}
$$

Remarque. On démontre sans difficulté que (7), (8) et (9) entraînent (2).

En précisant toutes les hypothèses nous supposons que la fonction cherchée $\sigma$ est définie dans le domaine (5) et que l'on a

$$
\sigma\left(x_{1}, x_{2}, x_{3}, x_{4}\right) \neq 0 \quad \text { pour }\left(x_{1}, x_{2}, x_{3}, x_{4}\right) \in D \text {. }
$$

Sous ces hypothèses nous allons résoudre le système (7)-(9) sans aucune hypothèse de régularité de la fonction $\sigma$. 
Nous partons de l'équation (9). Cette équation peut être réduite à l'équation de D. M. Sinzow [1], p. 156. Cette réduction nous fournit la solution sous la forme suivante

$$
\sigma\left(x_{1}, x_{2}, x_{3}, x_{4}\right)=\frac{\gamma\left(x_{1}, x_{2}, x_{3}\right)}{\gamma\left(x_{1}, x_{2}, x_{4}\right)},
$$

où la fonction inconnue $\gamma(x, y, z)$ est différente de zéro et définie pour $x \neq y \neq z \neq x$.

En tenant compte de la relation (11) nous obtenons d'après (7) et (8) les deux équations fonctionnelles suivantes pour la fonction $\gamma$ :

$$
\begin{gathered}
\frac{\gamma\left(x_{3}, x_{4}, x_{1}\right)}{\gamma\left(x_{3}, x_{4}, x_{2}\right)}=\frac{\gamma\left(x_{1}, x_{2}, x_{3}\right)}{\gamma\left(x_{1}, x_{2}, x_{4}\right)}, \\
\frac{\gamma\left(x_{1}, x_{2}, x_{3}\right)}{\gamma\left(x_{1}, x_{2}, x_{4}\right)}+\frac{\gamma\left(x_{1}, x_{3}, x_{2}\right)}{\gamma\left(x_{1}, x_{3}, x_{4}\right)}=1 .
\end{gathered}
$$

Introduisons quelques fonctions auxiliaires, notamment:

$$
A(x, y) \stackrel{\text { def }}{=} \gamma(x, 0, y), \quad B(x, y) \stackrel{\text { def }}{=} \gamma(x, y, 0) \quad \text { pour } x \neq y
$$

et

$$
a(x) \stackrel{\text { def }}{=} A(1, x), \quad \beta(x)=A(x, 1) \quad(x \neq 0,1) .
$$

En rappelant que $\gamma \neq 0$ on obtient de (12), en $y$ substituant $x_{2}=0$, et en changeant convenablement les notations -

$$
\gamma(x, y, z)=B(x, y) \cdot \frac{A(z, x)}{A(z, y)} .
$$

En substituant pareillement $x_{2}=0$ dans (13) on obtient

$$
\gamma(x, y, z)=\frac{B(x, y) \cdot A(x, z)}{A(x, z)-A(x, y)} .
$$

Remarquons que $A(x, z) \neq A(x, y)$ pour $z \neq y$. En effet, en posant dans (13) $x_{2}=0, x_{1}=x, x_{3}=y, x_{4}=z$ on obtient d'après l'hypothèse $A(x, z)=A(x, y) \quad \gamma(x, y, z)=0$, ce qui contredit à (10). Les formules (16) et (17) donnent

$$
\frac{A(x, z)}{A(z, y)}=\frac{A(x, z)}{A(x, z)-A(x, y)} .
$$

En substituant dans (18) $x=1$ nous obtenons d'après (15)

$$
\frac{\beta(z)}{A(z, y)}=\frac{a(z)}{a(z)-a(y)}
$$


d'où

$$
A(x, y)=\beta(x) \cdot \frac{\alpha(x)-\alpha(y)}{\alpha(x)} .
$$

La substitution de la dernière formule dans (17) nous fournit finalement

$$
\gamma(x, y, z)=B(x, y) \cdot \frac{a(x)-a(z)}{a(y)-\alpha(z)} .
$$

Cette formule est valable pour tous les $x, y, z$ différents et tels que $x, y, z \neq 0,1$.

La substitution de la formule (20) dans (17) donne

$$
\sigma\left(x_{1}, x_{2}, x_{3}, x_{4}\right)=\frac{\left[a\left(x_{1}\right)-a\left(x_{3}\right)\right] \cdot\left[a\left(x_{2}\right)-a\left(x_{4}\right)\right]}{\left[a\left(x_{2}\right)-a\left(x_{3}\right)\right] \cdot\left[a\left(x_{1}\right)-a\left(x_{4}\right)\right]}
$$

pour $x_{i} \neq x_{j}(i \neq j)$ et $x_{i} \neq 0,1$.

En posant dans la fonction $\gamma\left(x_{1}, x_{2}, x_{3}\right), x_{1}=3, x_{2}=2$ e'est-à-dire en définissant

$$
\tilde{a}(x) \stackrel{\text { det }}{=} \gamma(3,2, x), \quad x \neq 2,3
$$

nous arrivons à la formule

$$
\sigma\left(x_{1}, x_{2}, x_{3}, x_{4}\right)=\frac{\left[\tilde{a}\left(x_{1}\right)-\tilde{a}\left(x_{3}\right)\right] \cdot\left[\tilde{a}\left(x_{2}\right)-\tilde{a}\left(x_{4}\right)\right]}{\left[\tilde{a}\left(x_{2}\right)-\tilde{a}\left(x_{3}\right)\right] \cdot\left[\tilde{a}\left(x_{1}\right)-\tilde{a}\left(x_{4}\right)\right]}
$$

valable pour tous les $x_{i}$ différents et $x_{i} \neq 2,3$.

De cette façon nous obtenons l'identité

$$
\frac{\left[\tilde{a}\left(x_{1}\right)-\tilde{a}\left(x_{3}\right)\right] \cdot\left[\tilde{a}\left(x_{2}\right)-\tilde{a}\left(x_{4}\right)\right]}{\left[\tilde { a } ( x _ { 2 } - \tilde { a } ( x _ { 3 } ) ] \cdot \left[\tilde{a}\left(x_{1}-\tilde{a}\left(x_{4}\right)\right]\right.\right.}=\frac{\left[\alpha\left(x_{1}\right)-\alpha\left(x_{3}\right)\right] \cdot\left[\alpha\left(x_{2}\right)-a\left(x_{4}\right)\right]}{\left[\alpha\left(x_{2}\right)-\alpha\left(x_{3}\right)\right] \cdot\left[\alpha\left(x_{1}\right)-\alpha\left(x_{4}\right)\right]}
$$

valable pour $x_{i} \neq 0,1,2,3(i=1,2,3,4), x_{i} \neq x_{j}$ si $i \neq j$.

En y fixant les valeurs $x_{1}, x_{2}, x_{3}$ et en posant $x_{4}=x$ nous en tirons la relation

$$
\tilde{a}(x)=\frac{\lambda a(x)+\mu}{\nu a(x)+\varrho},
$$

$\lambda, \mu, \nu, \varrho$ étant des constantes. Cette relation nous permet d'étendre la définition de la fonction $a(x)$ en sorte que la formule (21) soit valable pour tous les quadruples $\left(x_{1}, x_{2}, x_{3}, x_{4}\right)$, où $x_{i}$ sont différents entre eux.

Inversement, on vérifie sans difficulté que, $a(x)$ étant une fonction quelconque, définie partout et inversible, le second membre de (21) représente une fonction satisfaisant aux équations fonctionnelles (7), (8), (9). On peut done énoncer le 
Thíorìme 1. La solution générale $\sigma$ du système d'équations (7)-(9) est donnée par la formule (21), a $(u)$ étant une fonction arbitraire inversible.

On peut maintenant posér le probleme suivant: sous quelles conditions supplémentaires le système (7)-(9) possède-t-il une solution unique

$$
\sigma\left(x_{1}, x_{2}, x_{3}, x_{4}\right)=\frac{\left(x_{1}-x_{3}\right)\left(x_{2}-x_{4}\right)}{\left(x_{2}-x_{3}\right)\left(x_{1}-x_{4}\right)}
$$

réproduisant le rapport anharmoniqueq Autrement dit: sous quelles conditions la fonction $a$ est-elle linéaire?

Nous allons résoudre cette question en faisant quelques hypothèses additionnelles.

Supposons d'abord que $\sigma$ soit une fonction continue par rapport à l'une des variables $x_{i}$. De la résulte que la fonction $a$ est continue et, comme $a$ est inversible, elle est strictement monotone et, par conséquent, dérivable sauf aux points d'un ensemble dénombrable $E$.

LEMmE. Si la fonction $\sigma$, définie dans $D$, satisfait aux équations (7)-(9) et si elle est continue par rapport à l'une des variables $x_{i}$, on a

$$
x \notin E, \quad a^{\prime} \neq 0 \rightarrow \lim _{h \rightarrow 0} \sigma(y, x, x-h, x+h)=-1
$$

pour chaque $y \neq x$, où $E$ est l'ensemble des points où a est dérivable.

Démonstration. Supposons $x \notin E$, c'est-à-dire admettons l'existence de la dérivée $\alpha^{\prime}(x)$. Nous avons d'après la formule (21)

$$
\begin{aligned}
\sigma(y, x, x-h, x+h) & =\frac{[a(y)-\alpha(x-h)] \cdot[a(x)-\alpha(x+h)]}{[\alpha(x)-\alpha(x-h)] \cdot[\alpha(y)-a(x+h)]} \\
& =\frac{\alpha(y)-\alpha(x-h)}{\alpha(y)-\alpha(x+h)} \cdot \frac{\alpha(x)-\alpha(x+h)}{\alpha(x)-a(x-h)} .
\end{aligned}
$$

$\alpha$ étant continue on a

$$
\lim _{h \rightarrow 0} \frac{\alpha(y)-a(x-h)}{\alpha(y)-\alpha(x+h)}=1 \quad \text { parce que } \alpha(y) \neq a(x)
$$

Nous transformons le deuxième facteur

$$
-\frac{\alpha(x)-a(x+h)}{\alpha(x)-\alpha(x-h)}=-\frac{\alpha(x+h)-\alpha(x)}{h}: \frac{\alpha(x-h)-a(x)}{-h} .
$$

Mais $\alpha$ possède au point $x$ une dérivée $\alpha^{\prime}(x) \neq 0$, donc le second membre de la formule précédente admet la limite $(-1)$. Nous avons done

$$
\lim _{h \rightarrow 0} \sigma(y, x, x-h, x+h)=-1
$$


Remarquons que dans le lemme précédent l'hypothèse $a^{\prime}(x) \neq 0$ est essentielle.

D'après le lemme précédent on peut dire que, si $h$ est petit la valeur

$$
\sigma(y, x, x-h, x+h)
$$

est, sous l'hypotèse de la continuité de la fonction $a$, égale à $(-1)$ pour. presque tous les points $x$ et pour tous les $y \neq x$ voisins de $x$; autrement dit, pour les quadruples $(y, x, x-h, x+h)$ le rapport anharmonique généralisé $\sigma$ est rapproché du rapport harmonique. Il se présente donc l'idée d'ajouter une hypothèse additionnelle postulant que pour les quadruples harmoniques d'une certaine classe la valeur de $\sigma$ soit égale à $(-1)$.

La totalité des quadruples $\left(x_{1}, x_{2}, x_{3}, x_{4}\right)$ harmoniques constitue une famille $F_{3}$ à trois paramètres essentiels. Nous allons envisager des sous-classes $F_{2}$ à deux paramètres ou bien $F_{1}$ à un paramètre. Pour fixer les idées nous définissons $F_{2}$ et $F_{1}$ respectivement comme il suit:

$$
\begin{aligned}
& F_{2} \stackrel{\text { def }}{=}\left\{\left(x_{1}, x_{2}, x_{3}, x_{4}\right) \mid x_{1}=x, x_{2}=y, x_{3}=\frac{2 x y}{x+y}, x_{4}=0\right\}, \\
& F_{1} \stackrel{\text { def }}{=}\left\{\left(x_{1}, x_{2}, x_{3}, x_{4}\right) \mid x_{1}=x, x_{2}=1, x_{3}=\frac{2 x}{x+1}, x_{4}=0\right\} .
\end{aligned}
$$

Le cas $F_{1}$ (plus difficile) sera le sujet d'une publication ultérieure.

Nous allons formuler ci-dessous un théorème où l'hypothèse concernant la régularité de la fonction $\alpha$ sera affaiblie. Observons notamment que, $\alpha$ étant inversible, l'hypothèse I: „a est strictement monotone” est plus faible que l'hypothèse II: „a est continue", paree que II implique directement I tandis que l'implication I $\Rightarrow$ II oblige de faire appel à l'équation fonctionnelle.

THEORÈME 2. Si la fonction a dans la formule

$$
\sigma\left(x_{1}, x_{2}, x_{3}, x_{4}\right)=\frac{\left[\alpha\left(x_{1}\right)-\alpha\left(x_{2}\right)\right] \cdot\left[\alpha\left(x_{2}\right)-\alpha\left(x_{4}\right)\right]}{\left[\alpha\left(x_{2}\right)-\alpha\left(x_{3}\right)\right] \cdot\left[\alpha\left(x_{1}\right)-a\left(x_{4}\right)\right]}
$$

est strictement monotone et si pour chaque quadruple harmonique $(x, y, 2 x y \mid x+y, 0)$ où $|x| \neq|y|$ (appartenant à la famille $\left.F_{2}\right)$ la valeur de $\sigma$ est égale à $(-1)$, alors la formule

a lieu.

$$
\sigma\left(x_{1}, x_{2}, x_{3}, x_{4}\right)=\frac{\left(x_{1}-x_{3}\right) \cdot\left(x_{2}-x_{4}\right)}{\left(x_{2}-x_{3}\right) \cdot\left(x_{1}-x_{4}\right)}
$$

Démonstration. En substituant $x_{1}=x, x_{2}=y, x_{3}=2 x y /(x+y)$, $x_{4}=0$ dans $\sigma\left(x_{1}, x_{2}, x_{3}, x_{4}\right)=-1$ nous obtenons

$$
\left[a(x)-\alpha\left(\frac{2 x y}{x+y}\right)\right] \cdot[a(y)-a(0)]=\left[a\left(\frac{2 x y}{x+y}\right)-\alpha(y)\right] \cdot[a(x)-a(0)]
$$


ou bien, en posant

$$
\begin{gathered}
\delta(x) \stackrel{\text { det }}{=} a(x)-a(0) \\
\delta\left(\frac{2 x y}{x+y}\right) \cdot[\delta(x)+\delta(y)]=2 \delta(x) \cdot \delta(y)
\end{gathered}
$$

Remarquons que $a$ étant, par hypothèse, strictement monotone, $\delta$ possède également la même propriété. L'égalité $\delta(0)=0$ entraine l'inégalité

$$
\delta(x) \neq 0 \quad \text { pour } x \neq 0 \text {. }
$$

Supposons pour le moment $x \neq 0$. Nous pouvons donc poser

$$
\gamma(x) \stackrel{\text { def }}{=} \frac{1}{\delta(1 / x)}
$$

et l'équation (27) prend la forme

$$
\gamma\left(\frac{x+y}{2 x y}\right)=\frac{1}{2}\left[\gamma\left(\frac{1}{x}\right)+\gamma\left(\frac{1}{y}\right)\right] .
$$

L'équation précédente veut dire que $\gamma(\xi)$ vérifie l'équation de Jensen (pour $\xi, \eta \neq 0$ au moins)

$$
\gamma\left(\frac{\xi+\eta}{2}\right)=\frac{1}{2}[\gamma(\xi)+\gamma(\eta)] .
$$

Mais on conclut de (29) que $\gamma$ est une fonction strictement monotone et, par conséquent, elle doit avoir la forme linéaire ([1], p. 49):

$$
\gamma(x)=\lambda x+\mu .
$$

La formule (31) n'est pas satisfaite, peut-être, pour 'x=0, mais, comme $\gamma$ est monotone, (31) subsiste pour tous les $x$.

En revenant aux fonctions $\delta$ et $a$ nous obtenons

$$
\begin{aligned}
& \delta(x)=\frac{1}{\gamma(1 / x)}=\frac{1}{(\lambda / x)+\mu}=\frac{x}{\lambda+\mu x}, \\
& a(x)=a(0)+\delta(x)=\frac{\tau+\omega x}{\lambda+\mu x},
\end{aligned}
$$

$\tau, \omega$ étant constantes. La fonction a résulte donc comme une fonction homographique. Remarquons cependant qu'une fonction homographique qui ne se réduit pas à une fonction linéaire $(\mu \neq 0)$ possède un point de discontinuité. Comme la fonction $\alpha$ doit être définie partout (à cause 
du domaine $D$ de la fonction $\sigma$ ), il suit que $\mu=0$ et, par conséquent, $a$ se réduit à une fonction linéaire ce qui établit la formule (23).

En terminant cette Note nous pourons énoncer le

THÉoǹ̀me 3. Etant donné le système (7)-(9), où $\sigma$ est défini dans (5) et satisfait à (10), la solution générale s'exprime par la formule (21), où $a(u)$ est une fonction inversible. Il y a donc une infinité de solutions. Pour obtenir la solution unique (23) il suffit de supposer que a est strictement monotone et en outre que $\sigma=-1$ pour chaque quadruple harmonique $\left(x, y, \frac{2 x y}{x+y}, 0\right)$ où $|x| \neq|y|$.

\section{Travaux citég}

[1] J. Aczél, Vorlesungen über Funktionalgleichungen und ihre Anwendungen, 1961. [2] -, S. Gołąb, M. Kuczma und E. Siwek, Das Doppelverhältnis als Lösung einer Funktionalgleichung, Ann. Polon. Math. 9 (1960), p. 183-187.

[3] W. Benz, Die 4-Punkt-Invarianten in der projektiven Geraden über einem Schiefkörper, ibidem 21 (1968), p. 97-101.

[4] - The n-point-invariants of the projective line and cross ratio of $n$-tuples, ibidem 26 (1972), p. 53-60.

[5] S. Topa, On a generalization of the functional equation for the harmonic ratio of four points on a projective line over an arbitrary commutative field, ibidem 23 (1970), p. 65-72. 